\title{
ON A THEOREM OF GOFFMAN CONCERNING SCHAUDER SERIES
}

ROBERT E. ZINK

1. A classical question posed by Lusin asks whether it is possible to find for each measurable function defined on $[0,2 \pi]$ a corresponding trigonometric series, with coefficients converging to zero, that converges almost everywhere to the function. This question was answered affirmatively by Men'šov [4] for real-valued functions, but the general question remains unanswered. Thus, it is of interest to inquire whether there be any Schauder bases for $L^{p}, p>1$, with respect to which every measurable function has a pointwise representation. Although Talalyan and Arutyanyan [8] have shown that a prime candidate, the Haar system, does not have this property, Gundy [3] has proved that systems of the specified type do exist.

The Schauder functions are total in each of the $L^{p}$ spaces, although they in no case constitute a basis. In [1], Goffman solved Lusin's problem for this system by way of a sequence of careful estimates culminating in a construction of the required series. An interesting aspect of this work is that not all of the Schauder functions are required for the construction. Indeed, it is clear from a superficial examination of the arguments employed in [1] that any finite number of functions could be deleted from the system and the work carried through with no resulting essential modification of the demonstrations.

In analogy with work of Talalyan [6], and Goffman and Waterman [2], it is appropriate to ask whether infinitely many Schauder functions could be discarded in such a way that the above-mentioned result of Goffman would remain in force for the abbreviated system. In the present note it is shown that this is the case, and a characterization is given of those subsystems for which the Goffman theorem holds. The result suggests that there may be lurking in the background some very general form of the Müntz theorem.

2. The Schauder system herein discussed is the usual collection of spike functions whose supports are dyadic intervals (see, for example, [1]). As it happens, each of these functions can be expanded (in an infinity of different ways) in an almost everywhere convergent series of the others. This fact is the key to the present discussion.

Received by the editors August 19, 1968. 
LеммA 1. Let $\Phi=\left\{\phi_{1}, \phi_{2}, \cdots\right\}$ be an infinite subsystem of the Schauder system, and let $\left\{E_{n}\right\}_{n=1}^{\infty}$ be the sequence of supports of the elements of $\Phi$. If

$$
\mu\left(\lim \sup _{n} E_{n}\right)=1
$$

then every Schauder function, $f$, can be expressed as a series $\sum_{n=1}^{\infty} a_{n} \phi_{n}$ converging a.e. to $f$, wherein $0 \leqq a_{n} \leqq 1$ and $\sum_{k=1}^{n} a_{k} \phi_{k} \leqq f$, for every $n$. (It is clear that $\left(^{*}\right)$ is also a necessary condition for the existence of such series.)

Proof. Let $f$ be a Schauder function not contained in $\Phi$ and let $E=\{x: f(x)>0\}$. Because $\left\{E_{n}: E_{n} \subset E\right\}$ constitutes a Vitali covering of $E$, it is possible to choose pairwise disjoint supports, $E_{n_{1}}, \cdots$, $E_{n_{k(1)}}$, such that $\mu\left(E \backslash \bigcup_{i=1}^{k(1)} E_{n_{i}}\right)<\frac{1}{4}$ and each $E_{n_{i}}$ is a subset of $E$. Denote the midpoint of $E_{n_{i}}$ by $x_{i}$, let

$$
\phi^{(1)}=f_{1}=\sum_{i=1}^{k(1)} f\left(x_{i}\right) \phi_{n_{i}},
$$

let $E^{(1)}=\bigcup_{i=1}^{k(1)} E_{n_{i}}$, and let $F^{(1)}=E \backslash E^{(1)}$. Then $f-f_{1} \geqq 0$, and

$$
\begin{aligned}
\int_{0}^{1}\left(f-f_{1}\right) d \mu & =\int_{E}=\int_{E}+\int_{F^{(1)}}<\frac{1}{4}+\frac{1}{2} \int_{E^{(1)}} f d \mu \\
& \leqq \frac{1}{4}+\frac{1}{2} J
\end{aligned}
$$

where $J=\int_{E} f d \mu=\|f\|_{1}$.

Let $E^{(1)}=G_{1}^{(1)} \cup \cdots \cup G_{N_{1}}^{(1)}$, where the $G_{k}^{(1)}$ are nonoverlapping (dyadic) intervals, such that, for each $k, f_{1}$ is linear over $G_{\mathfrak{k}}^{(1)}$, vanishes at one end of $G_{k}^{(1)}$ and coincides with $f$ at the other. From an application of the Vitali theorem to $V_{1}=\left\{E_{n}: n>n_{k(1)}\right\}$ follows the existence of a finite subfamily of disjoint members of $v_{1}$, each element of which is contained in some $G_{k}^{(1)}$ and whose union covers $E^{(1)}$, save for a set of measure less than $1 / 32$. Proceeding in the same manner, let $F^{(1)}$ $=H_{1}^{(1)} \cup \ldots \cup H_{M_{1}}^{(1)}$, where the $H_{\mathbf{k}}^{(1)}$ are nonoverlapping (dyadic) intervals, such that $f$ is linear over each of them. Again there are finitely many pairwise disjoint elements of $V_{1}$, each of which lies entirely within some $H_{\mathbf{k}}^{(1)}$, whose union covers $F^{(1)}$, save for a set of measure less than $1 / 16$.

Let $\left\{E_{n_{k(1)+1}}, \cdots, E_{n_{k(2)}}\right\}$ be the union of the two subsets of $v_{1}$ thus selected, let $E^{(2)}=\bigcup_{i=k(1)+1}^{(2)} E_{n_{i}}$, and let $F^{(2)}=E \backslash E^{(2)}$. Again denoting the center of $E_{n_{i}}$ by $x_{i}$, let 


$$
\phi^{(2)}=\sum_{i=k(1)+1}^{k(2)}\left(f-f_{1}\right)\left(x_{i}\right) \phi_{n_{i}},
$$

and let $f_{2}=\phi^{(1)}+\phi^{(2)}$. Then $0 \leqq f_{1} \leqq f_{2} \leqq f$, and

$$
\begin{aligned}
& \int_{0}^{1}\left(f-f_{2}\right) d \mu=\int_{F^{(2)} \cap^{(1)}}+\int_{E^{(2)} \cap^{(1)}}+\int_{F^{(2)} \cap^{(1)}}+\int_{E}^{(2)} \cap^{(1)} \\
& <\frac{1}{16}+\frac{3}{32}+\frac{1}{32}+\frac{1}{2^{2}} J \\
& =\frac{1}{4}\left(\frac{3}{4}\right)+\frac{1}{2^{2}} J \text {. }
\end{aligned}
$$

Proceeding thus inductively, one constructs for every natural number $n$ a finite Schauder series

$$
f_{n}=\sum_{i=1}^{k(n)} a_{i} \phi_{n_{i}}
$$

such that

$$
0 \leqq \sum_{i=1}^{j} a_{i} \phi_{n_{i}} \leqq \sum_{i=1}^{j+1} a_{i} \phi_{n_{i}} \leqq f \quad(j),
$$

$0 \leqq a_{i} \leqq 1$, for all $i$, and

$$
\int_{0}^{1}\left(f-f_{n}\right) d \mu<\frac{1}{4}\left(\frac{3}{4}\right)^{n-1}+\frac{1}{2^{n}} J \quad(n) .
$$

From an application of the dominated convergence theorem, it follows that

$$
f(x)=\sum_{i=1}^{\infty} a_{i} \phi_{n_{i}}(x) \quad \text { a.e. }
$$

3. There follows an outline of a constructive scheme that leads to the result promised in the introduction. Although the work could be carried out directly, it seems most simple to stand on the shoulders of Goffman.

LEMMA 2 (GoFFMAN). Let $f$ be an a.e.-real-valued measurable function, let $\epsilon$ be a positive number, and let $m$ be a natural number. If $E$ $=\{x:|f(x)| \leqq \epsilon\}$ and if $\eta$ is any positive number, then there is a Schauder expansion $\sum_{i=m}^{n} a_{i} x_{i}$ such that all coefficients do not exceed $\epsilon+\eta$ in absolute value, 


$$
\left|\sum_{i=m}^{j} a_{i} x_{i}(t)\right| \leqq \epsilon \quad(m \leqq j \leqq n),
$$

for every $t$ in $D$, where $D$ is a subset of $E$ having measure greater than $\mu(E)-\eta$, and

$$
\left|f(t)-\sum_{i=m}^{n} a_{i} x_{i}(t)\right| \leqq \eta,
$$

for every $t$ in $H$, where $\mu(H)>1-\eta$.

THEOREM 3. If $\Phi=\left\{\phi_{1}, \phi_{2}, \cdots\right\}$ is a subsystem of the Schauder system for which condition (*) is satisfied, then every extended-realvalued measurable function defined on $[0,1]$ can be expanded in an almost everywhere convergent $\Phi$-series, with sequence of expansion coefficients converging to zero.

Proof. Suppose first that $f$ is a real-valued measurable function.

Let $\left\{\epsilon_{n}\right\}_{n=1}^{\infty}$ be a sequence of positive numbers such that $\sum_{n=1}^{\infty} \epsilon_{n}$ converges. From the lemma of Goffman, one knows that there is a finite Schauder series $\sum_{i=1}^{m(1)} a_{i} x_{i}$ such that

$$
\left|f(t)-\sum_{i=1}^{m(1)} a_{i} x_{i}(t)\right|<\epsilon_{1}
$$

for all $t$ in a set $E_{1}$ with $\mu\left(E_{1}\right)>1-\epsilon_{1}$. By virtue of Lemma 1, one may replace $\sum_{i=1}^{m(1)} a_{i} x_{i}$ by $\sum_{i=1}^{n(1)} b_{i} \phi_{i}$ while retaining the same estimate.

Let $f_{1}=f-\sum_{i=1}^{n(1)} b_{i} \phi_{i}$. Again, according to Goffman, there is a finite Schauder series $\sum_{i=1}^{m(2)} a_{i}^{(2)} x_{i}$ such that $\left|a_{i}^{(2)}\right| \leqq \epsilon_{1}+\epsilon_{2}$, for each $i,\left|\sum_{i=1}^{j} a_{i}^{(2)} x_{i}(t)\right|<\epsilon_{1}$, for all $j \leqq m(2)$ and for all $t$ in $H_{1}^{*}$, where $\mu\left(H_{1}^{*}\right)>1-\epsilon_{1}-\epsilon_{2} / 2$, and

$$
\left|f_{1}(t)-\sum_{i=1}^{m(2)} a_{i}^{(2)} x_{i}(t)\right|<\frac{\epsilon_{2}}{2},
$$

for all $t$ in $E_{2}^{*}$, with $\mu\left(E_{2}^{*}\right)>1-\epsilon_{2} / 2$.

Now apply Lemma 1 to the function $x_{1}$, using $\Phi_{1}=\Phi \backslash\left\{\phi_{i}: i \leqq n(1)\right\}$ in place of $\Phi$ (the system $\Phi_{1}$ also possesses property $\left({ }^{*}\right)$ ), in order to obtain a finite $\Phi$-series $\sum_{i=n(1)+1}^{n(1)+k(1)} b_{i}^{*} \phi_{i}$, with coefficients between 0 and 1 , such that

$$
\left|x_{1}(t)-\sum_{i=n(1)+1}^{n(1)+k(1)} b_{i}^{*} \phi_{i}(t)\right|<\frac{\epsilon_{2}}{2[1+m(2)]\left(\epsilon_{1}+\epsilon_{2}\right)}
$$

on a set $K_{11}$ having measure exceeding $1-\epsilon_{2} / 2[1+m(2)]$. In similar 
fashion approximate $x_{2}$ by a finite $\Phi$-series $\sum_{i=n(1)+k(1)+1}^{n(1)+k(2)} b_{i}^{*} \phi_{i}$ such that

$$
\left|x_{2}(t)-\sum_{i=n(1)+k(1)+1}^{n(1)+k(2)} b_{i}^{*} \phi_{i}(t)\right|<\frac{\epsilon_{2}}{2[1+m(2)]\left(\epsilon_{1}+\epsilon_{2}\right)}
$$

on a set $K_{12}$ having measure exceeding $1-\epsilon_{2} / 2[1+m(2)]$, the coefficients again lying between 0 and 1 , and continue thus to approximate each of the $x_{i}$ involved in the Goffman approximation of $f_{1}$.

For $n(1)+k(j)<i \leqq n(1)+k(j+1)$ let $b_{i}=a_{j+1}^{(2)} b_{i}^{*}, 0 \leqq j \leqq m(2)-1$ $(k(0)=0)$. There results a $\Phi$-polynomial $\sum_{i-n(1)+1}^{n(2)} b_{i} \phi_{i}$ having the following properties:

(1) $\left|b_{i}\right| \leqq \epsilon_{1}+\epsilon_{2}(n(1)+1 \leqq i \leqq n(2))$,

(2) $\left|\sum_{i=n(1)+1}^{j} b_{i} \phi_{i}\right| \leqq 2\left(\epsilon_{1}+\epsilon_{2}\right)$, on $H_{1}=H_{1}^{*} \cap \bigcap_{k=1}^{m(2)} K_{1 k}(n(1)$ $+1 \leqq j \leqq n(2))$,

(3) $\left|f_{1}(t)-\sum_{i=n(1)+1}^{n(2)} b_{i} \phi_{i}(t)\right|<\epsilon_{2}$, for all $t$ in $E_{2}=D_{2}^{*} \cap \bigcap_{k=1}^{m(2)} K_{1 k}$. Note that the series has been cleverly chosen so that $\mu\left(E_{2}\right)>1-\epsilon_{2}$.

For the next step in the construction process, there is, according to Lemma 2, an approximation $\sum_{i=1}^{m(3)} a_{i}^{(3)} x_{i}$ to $f_{2}=f_{1}-\sum_{i=1}^{n(2)} b_{i} \phi_{i}$ such that each $a_{i}^{(3)}$ has modulus not greater than $\epsilon_{2}+\epsilon_{3}$,

$$
\left|\sum_{i=1}^{j} a_{i}^{(3)} x_{i}(t)\right| \leqq \epsilon_{2} \quad(i \leqq j \leqq m(3)),
$$

for every $t$ in $H_{2}^{*}$, with $\mu\left(H_{2}^{*}\right)>1-\epsilon_{2}-\epsilon_{3} / 2$, and

$$
\left|f_{2}(t)-\sum_{i=1}^{j} a_{i}^{(3)} x_{i}(t)\right|<\frac{\epsilon_{3}}{2}
$$

on $E_{3}^{*}$, where $\mu\left(E_{2}^{*}\right)>1-\epsilon_{3} / 2$. Proceeding exactly as before, one obtains a $\Phi$-polynomial $\sum_{i=n(2)+1}^{n(3)} b_{i} \phi_{i}$ such that:

(1) $\left|b_{i}\right| \leqq \epsilon_{2}+\epsilon_{3} \quad(n(2)+1 \leqq i \leqq n(3))$,

(2) $\left|\sum_{i=n(2)+1}^{j} b_{i} \phi_{i}(t)\right|<2\left(\epsilon_{2}+\epsilon_{3}\right)$ on $H_{2}$, with $\mu\left(H_{2}\right)>1-\epsilon_{2}-\epsilon_{3}$ $(n(2)+1 \leqq j \leqq n(3))$,

(3) $\left|f_{2}(t)-\sum_{i=n(2)+1}^{n(3)} b_{i} \phi_{i}(t)\right|<\epsilon_{3}$ on $E_{3}$, with $\mu\left(E_{3}\right)>1-\epsilon_{3}$.

Continuing inductively, one finds an increasing sequence of natural numbers $\{n(k)\}_{k=1}^{\infty}$ and sequences of measurable sets, $\left\{H_{k}\right\}_{k=1}^{\infty}$, $\left\{E_{k}\right\}_{k=1}^{\infty}$, such that, for every $k$ :

(a) $\mu\left(H_{k}\right)>1-\epsilon_{k}-\epsilon_{k+1}$ and $\mu\left(E_{k}\right)>1-\epsilon_{k}$,

(b) there is a series $\sum_{i=n(k)+1}^{n(k+1)} b_{i} \phi_{i}$ satisfying:

(i) $\left|b_{i}\right| \leqq \epsilon_{k}+\epsilon_{k+1}(n(k)+1 \leqq i \leqq n(k+1))$,

(ii) $\left|\sum_{i=n(k)+1}^{j} b_{i} \phi_{i}(t)\right|<2\left(\epsilon_{k}+\epsilon_{k+1}\right)(n(k)+1 \leqq j \leqq n(k+1))$, on $H_{k}$, 
(iii) $\left|f_{k}(t)-\sum_{i=n(k)+1}^{n(k+1)} b_{i} \phi_{i}(t)\right|<\epsilon_{k+1}$ on $E_{k+1}$, where $f_{k}=$ $f_{k-1}-\sum_{i=n(k-1)+1}^{n(k)} b_{i} \phi_{i}$.

In order to demonstrate the a.e. convergence of $\sum_{i=1}^{\infty} b_{i} \phi_{i}$ to $f$, note that, for every $k$,

$$
f=f_{k}+\sum_{i=1}^{n(k)} b_{i} \phi_{i}
$$

so that for every $j>n(k+1)$,

$$
\begin{aligned}
\left|f(t)-\sum_{i=1}^{j} b_{i} \phi_{i}(t)\right| & =\left|f_{k}(t)-\sum_{i=n}^{j} b_{i} \phi_{i}(t)\right| \\
& <\epsilon_{k+1}+2 \sum_{i=k+1}^{\infty}\left(\epsilon_{i}+\epsilon_{i+1}\right)
\end{aligned}
$$

for all $t$ in $E_{k+1} \cap \bigcap_{i=k+1}^{\infty} H_{i}$. Because the measure of this set exceeds $1-3 \sum_{i=k+1}^{\infty} \epsilon_{i}$, the convergence is established.

In the general case, following Goffman, let $f=f_{1}+f_{2}+f_{3}$, where $f_{1}$ is finite, $f_{2}$ is $+\infty$ on a measurable set $A$ and 0 on the complement of $A$, and $f_{3}$ is $-\infty$ on a measurable set $B$ and 0 on the complement of $B$. A trivial modification of an argument of Goffman shows that there are $\Phi$-series $\sum_{i=1}^{\infty} a_{i} \phi_{i}$ and $\sum_{i=1}^{\infty} b_{i} \phi_{i}$, with coefficient sequences converging to zero, the one series converging to $+\infty$ a.e. on $A$ and to a real-valued function a.e. on $[0,1] \backslash A$, the other converging to $-\infty$ a.e. on $B$ and to a real-valued function a.e. on $[0,1] \backslash B$. If the functions to which these series converge be designated by $g_{2}$ and $g_{3}$, then let $\sum_{i=1}^{\infty} c_{i} \phi_{i}$ be an a.e.-convergent representation (with $\left.\lim _{n} c_{n}=0\right)$ of the real function $f_{1}-g_{2} \chi_{[0,1] \backslash A}-g_{3} \chi_{[0,1] \backslash B}$. The series $\sum_{i=1}^{\infty}\left(a_{i}+b_{i}+c_{i}\right) \phi_{i}$ has the required properties.

4. In a paper dealing primarily with families of Haar functions [5], J. J. Price and the author observed that a family of Schauder functions is total in measure if the condition $\left(^{*}\right)$ (above) is satisfied. That theorem (Theorem 11 of [5]) is an immediate consequence of Lemma 1 . Indeed, it is worthy of note that a family satisfying $\left(^{*}\right)$ is total in each of the spaces $L^{p}[0,1], 1 \leqq p<\infty$, as the construction process of Lemma 1 makes clear.

(In this general connection, Theorem 11 does not tell the right story. For, in analogy with the work of Talalyan [7], one should expect that making certain deletions from the Schauder system (a Schauder basis for $C[0,1]$ ) would yield a subsystem still total in each of the spaces of continuous functions over suitable measurable subsets of $[0,1]$ having measure arbitrarily close to 1 . Such a proposition is an immediate consequence of Theorem 11, of course.) 
One final comment concerning Lemma 1 is in order. The following remark is reminiscent of a classical result of Müntz.

Corollary 4. A subsystem $\left\{x_{n_{i}}: i=1,2, \cdots\right\}$ of the Schauder system is sufficient for pointwise representation of all measurable functions only if $\sum_{i=1}^{\infty} 1 / n_{i}$ diverges.

Proof. By virtue of Lemma 1, $\sum_{i=1}^{\infty} \mu\left(E_{n_{i}}\right)=+\infty$. Divide the Schauder system into subsets, the members of which have supports of equal measure. If the natural ordering be employed, then for $n$ greater than 2 , the members of the $n$th subset of this partition have subscripts of the form $2^{n-2}+j$, with $0 \leqq j<2^{n-2}$. Suppose that there are $k_{n}$ elements of the sequence $\left\{n_{i}\right\}_{i=1}^{\infty}$ for which $x_{n_{i}}$ belongs to the $n$th subset of the given partition. Since for each $j$ satisfying $0 \leqq j<2^{n-2}$, $1 /\left(2^{n-2}+j\right) \geqq 1 / 2^{n-1}$, it follows that

$$
\begin{aligned}
\sum_{i=1}^{\infty} \frac{1}{n_{i}} & \geqq \sum_{n=3}^{\infty} \frac{k_{n}}{2^{n-1}} \\
& =2^{r} \sum_{i=8}^{\infty} \mu\left(E_{n_{i}}\right)=+\infty,
\end{aligned}
$$

where $r$ and $s$ could be determined if one had the patience.

The condition obviously is not sufficient; the collection of Schauder functions whose supports lie in $\left[0, \frac{1}{2}\right]$ furnishes a simple counterexample.

\section{REFERENCES}

1. Casper Goffman, Remark on a problem on Lusin, Acta Math. 111 (1964), 63-72.

2. Casper Goffman and Daniel Waterman, Basic sequences in the space of measurable functions, Proc. Amer. Math. Soc. 11 (1960), 211-213.

3. Richard F. Gundy, Martingale theory and pointwise convergence of certain orthogonal series, Trans. Amer. Math. Soc. 124 (1966), 228-248.

4. D. E. Men'šov, Sur la represéntation des fonctions mesurables des séries trigonométriques, Mat. Sb. 9 (1941), 667-692.

5. J. J. Price and Robert E. Zink, On sets of completeness for families of Haar functions, Trans. Amer. Math. Soc. 119 (1965), 262-269.

6. A. A. Talalyan, On the convergence almost everywhere of subsequences of partial sums of general orthogonal series, Izv. Akad. Nauk Armjan. SSR Ser. Fiz.-Mat. Nauk 10 (1957), 17-34. (Russian. Armenian summary)

7. - Representation of measurable functions by series, Uspehi Mat. Nauk 15 (1960), 77-142 = Russian Math. Surveys 15 (1960), 75-136.

8. A. A. Talalyan and F. G. Arutyanyan, On convergence of Haar series to $+\infty$, Mat. Sb. 66 (1965), 240-247. (Russian)

Purdue University 